\title{
Traditional Cultural Short Video Publicity System Based on Data Mining and Digital Image Technology
}

\author{
Xiaoyue Guo ${ }^{1}$ and Jingjing Feng $\mathbb{D}^{2}$ \\ ${ }^{1}$ Qiyue Media College, Cangzhou Normal University, Cangzhou 061000, China \\ ${ }^{2}$ Personnel Office, Hebei Vocational College of Rail Transportation, Shijiazhuang 050051, China
}

Correspondence should be addressed to Jingjing Feng; p39@wanfeng.edu.bi

Received 14 October 2021; Revised 6 November 2021; Accepted 20 November 2021; Published 11 December 2021

Academic Editor: Qiangyi Li

Copyright (C 2021 Xiaoyue Guo and Jingjing Feng. This is an open access article distributed under the Creative Commons Attribution License, which permits unrestricted use, distribution, and reproduction in any medium, provided the original work is properly cited.

\begin{abstract}
With the continuous development of the social economy, more and more attention has been paid to traditional cultures, such as Chinese-style clothing, decoration, and furniture, and the publicity of traditional culture is also diverse. However, it should be noted that the rise of short videos has promoted the diversification of traditional culture publicity methods. In this paper, data mining technology and digital technology are introduced. By sorting out the content, publicity strategy, and emphasis of traditional culture, and through correlation analysis, the audience of traditional culture has been wider, and the content has been transmitted more, aiming to make more Chinese people love traditional culture and promote traditional culture. The simulation results show that data mining and digital technology are effective and can effectively promote the propaganda of short videos of traditional culture.
\end{abstract}

\section{Introduction}

With the continuous development of the social economy, traditional culture is more and more favored by people, such as Chinese furniture, songs, and clothes [1,2]. The development of digitalization and information technology has promoted the continuous enrichment of the expression forms of traditional culture, such as AR, VR, and short videos. On the one hand, more people can understand the traditional culture, such as the existing intangible cultural heritage, and on the other hand, it also reflects the protection and reuse of traditional culture by the country $[3,4]$. Intangible cultural heritage culture, as a special traditional culture, has gradually formed layered, comprehensive, and diverse cultural values in the process of practical application, which has ornamental value and economic value at the same time. It is precisely because of these special attributes and cultures that more Chinese people are keen on traditional culture and also gradually set off the pursuit of traditional culture publicity and digital forms of expression [5-7]. For example, Mr. Fang Jinlong can play different music sounds on a lute, which promotes the essence of Chinese traditional culture. For example, the underwater Chinese wind launched by Henan Satellite TV reflects the aestheticism and beauty of traditional Chinese culture.

In the information age, the construction and development of various short videos and information systems have promoted the dissemination and development of traditional culture to a certain extent $[8,9]$. But it is worth the attention that the traditional culture accumulated a lot of information behind, that is, abundant data. If not for timely and effective processing, the data will explode. The growth of these data cannot bring the accumulation of knowledge because of excessive invalid data. It is therefore not possible to extract effective information from existing data, such as recommending the corresponding traditional cultural Chinese wind song. These need more detailed recommendations to better support digital traditional cultural expression forms. In the actual application process, data mining technology needs to be introduced to extract corresponding knowledge so as to provide decision-making support for traditional culture [10]. 
According to these limitations and demands, this paper introduced data mining technology and digital technology through combing the content of traditional culture, traditional culture from the viewpoint of short video propaganda, and analysis of how to innovate on technology. These data are sufficient for data cleaning and data mining, to provide a more accurate knowledge. It aims to promote traditional culture from the perspective of new media.

\section{Share the Overall Data Center Framework}

For the new media conditions of traditional culture, the data for normal short video propaganda are very important, especially with the continuous development of mobile terminals. Because of this, everyone can use the terminal to use the traditional culture of short videos for the corresponding videos, pictures, etc., but the information and multifarious data do not implement effective knowledge organization. Moreover, there is no better filtering and data sharing $[11,12]$.

As for the user's terminal, it is not the data source of traditional cultural data. It is updated regularly by the fixed traditional culture team, transferred through the data center, and pushed to the hands of each user. The user may only have browsing permission and cannot modify, but they can re-create the process.

Data sharing does not just reflect data information browsing; more from the system, the comprehensive utilization of various terminals for data; therefore, the need for data cleaning, pretreatment, mining, shared data hungry, analysis, and knowledge extraction helps choose the appropriate method to build the rules of data mining, and contact the actual situation. It can also provide corresponding help for managers or decision makers $[13,14]$.

2.1. Overview of Decision Tree Algorithm. As for the decision tree, it is essentially a learning algorithm of induction and summary, which is a classification rule of the expression form of the decision tree formed by calculation and synthesis from a series of unorganized, unordered, and irregular data groups. Generally, a decision tree is composed of nodes, branches, and leaves. Each node corresponds to each attribute. A leaf represents the corresponding category, and the top layer of the tree represents the corresponding root node. The typical methods of the decision tree are more, from the root node to the leaf node, forming each path classification rule. The decision tree method is usually used to enhance the information of the attribute or other tree structure through the study of the reduction of redundant information, realize the continuous attributes of data processing, and form the rule of incomplete treatment $[15,16]$.

2.2. Data Acquisition and Preprocessing. The research in this paper mainly adopts the relationship between the basic evaluation of traditional culture and the evaluation results. By establishing a corresponding evaluation model for traditional culture, experts are encouraged to have a clear evaluation model. The specific data are shown in Figures 1 and 2.

2.3. Quality Evaluation Model Based on the C4.5 Decision Tree. Firstly, an appropriate decision tree model and algorithm are selected to evaluate the publicity quality of short videos of traditional culture in 2020. Specifically, 4000 evaluation data are mined to extract the corresponding classification rules which are as follows:

Rule 1: IF educational background = Doctoral degree AND professional title $=$ professor AND age W50THEN the proportion of propaganda quality evaluation of traditional culture short video is "excellent" is $88.3 \%$

Rule 2: IF educational background=master's degree AND professional title $=$ engineer AND age $>30$ THEN the proportion of propaganda quality evaluation of short videos of traditional culture is "excellent" is $84.6 \%$

Rule 3: IF education = undergraduate AND professional title $=$ assistant engineer AND age W25 The proportion of traditional culture short video publicity quality evaluation is "excellent" is $91.6 \%$

\subsection{Interpretation and Practical Application of the Rules.} As can be seen from the classification rules, more young people love traditional culture and prefer to promote it in the form of short videos, so as to realize the reuse, reprocessing, and redisplay of traditional culture.

For the mapping model of association rules, through the classification of uniform rules for building, the first is the father and son node level classification, which is geared to the needs of complex relationships, not simply defined by the relationship between the father and the son node directly. The need to build a more complex mapping model reaction and, at the same time, using the hierarchical relations are defined, combined with the actual demand. The corresponding mapping model is constructed and assumed.

Definition 1. Set the corresponding association rule mapping model as a tuple, defined by five factors.

Firstly, the corresponding mapping model concept book is constructed, and the specific data-based relationship is shown in Figure 3.

After building the corresponding mapping model, it is necessary to carry out associated mapping to the data, followed by data analysis, to build a one-to-one mapping relationship and realize the correlation and mapping between data structure and data.

Definition 2. Set the corresponding associated mapping model, use the mapping function for automatic mapping, and set the corresponding set, where

(1) Initialize the concept set of association rules, and make it clear that it is an infinite set

(2) Initialize the relation set in the association rule, which is also an infinite set 


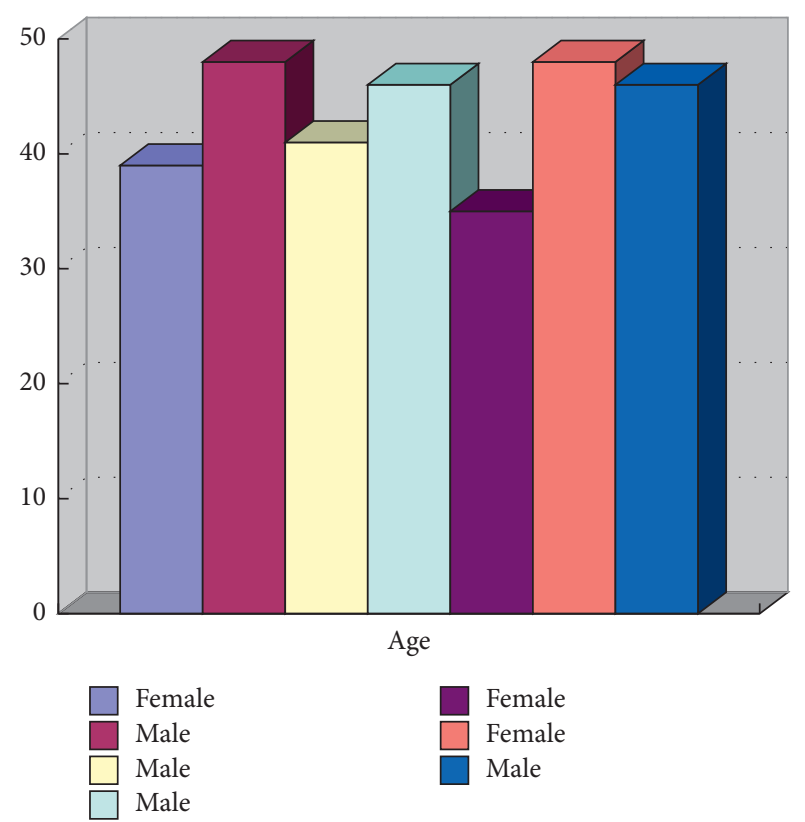

Figure 1: Basic information of evaluators of traditional culture short video publicity.

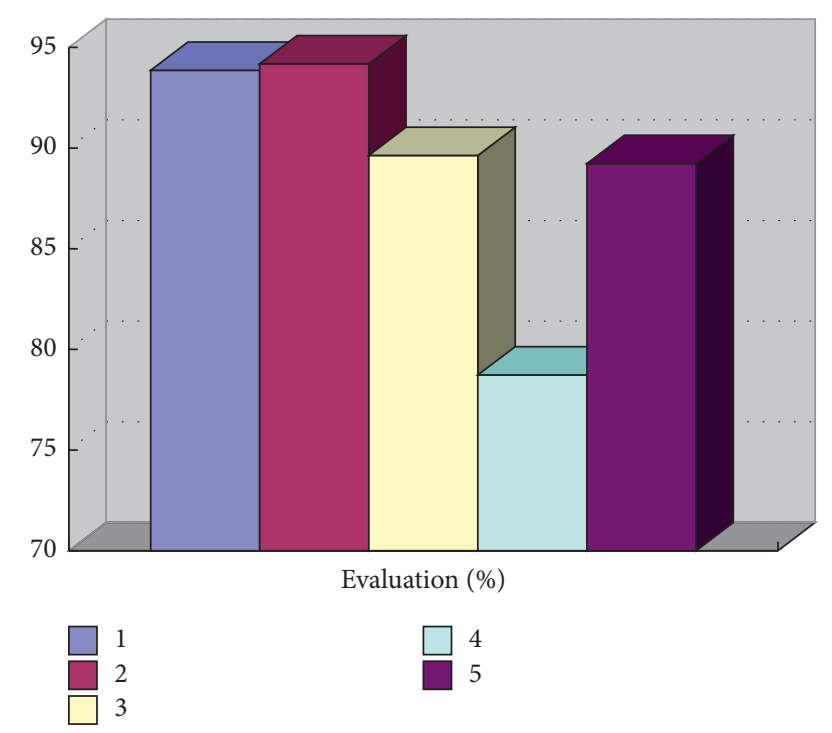

Figure 2: Evaluation results of short videos of traditional culture.

For the mapped database node, it performs overall filtering through different expressions to realize the efficiency of keyword retrieval and improve the efficiency of associated results. The specific process is shown in Figure 4 .

Corresponding association rule data mining is characterized by finding association rule relations between minimum confidence and minimum support according to attribute items $[17,18]$.

If $A$ and $B$ are item sets, $A \subset I$ and $B \subset I$, and dynamic association rules can be calculated using formula (1), as shown as follows:

$$
S(A \cup B)_{i}=\frac{f_{(A \cup B)_{i}}}{\left|D_{i}\right|}, \quad(i \in\{1,2, \ldots, n\}),
$$

where $f_{(A \cup B)}$ is the number of $A \cup B$ occurrences in the data subset $D_{i}(i \in\{1,2, \ldots, n\})$, and $\left|D_{i}\right|$ is the number of things in $D_{i}$. If the support degree of rule $A \Rightarrow B$ is set as $S$, formula (2) can be used for calculation, as shown as follows:

$$
S=S_{(A \cup B)}=\frac{f_{(A \cup B)}}{M}=\sum_{i=1}^{n} \frac{f_{(A \cup B)_{i}}}{M},
$$

where $M$ is the number of transactions in $D$.

\section{How to Innovate Traditional Cultural Content in Short Video Platform}

As for short videos, they have become an emerging form of expression in recent years, especially in the current popular mobile devices. Using short videos to carry out traditional cultural innovation is one of the most important forms of expression. On the one hand, original short videos can retain the authenticity of traditional culture and avoid the effects of documentaries. On the other hand, real traditional culture can be boldly expressed through the combination of virtual and reality, interesting culture and various carriers, so that more people can understand traditional culture, production technology, and important history and culture.

But for a short video platform, to carry out publicity and innovation of traditional culture form, two ways can be followed: on the one hand, in the form of a short video to show and give an audience broader, more traditional cultural and more rich content; on the other hand, it can retain more traditional culture carriers, better and more excellent records of the implementation of the culture, preventing the cultural discontinuity and disappearance.

The use of new media platforms focused on the form of the traditional culture helps carry on the important feature of the process; the content is more intuitive and decreases the difficulty of the corresponding to imitate its specific content. This makes the traditional culture making process more relaxed and more intuitive, further cause the enthusiasm of the audience, which in turns increase the love of the traditional culture. At the same time, it strengthens the spread of the traditional cultural character, highlighting the fun of traditional culture.

In addition, the use of new media short videos can also introduce traditional culture to the campus and help pass the concept of traditional culture to the next generation.

\subsection{Content Fragmentation and Typification of Short Video} Platform. For the moment, short video content is too fragmented and typifying too. Although there is a lot of hanfu, or Chinese-style furniture to let more foreigners, foreigners can understand the corresponding Chinese traditional culture, including Chinese kung fu, but for deeper level understanding, the historical origin is difficult to contact. Therefore, for the short video advertising, it should focus more on its real connotation, rather than simply push, 


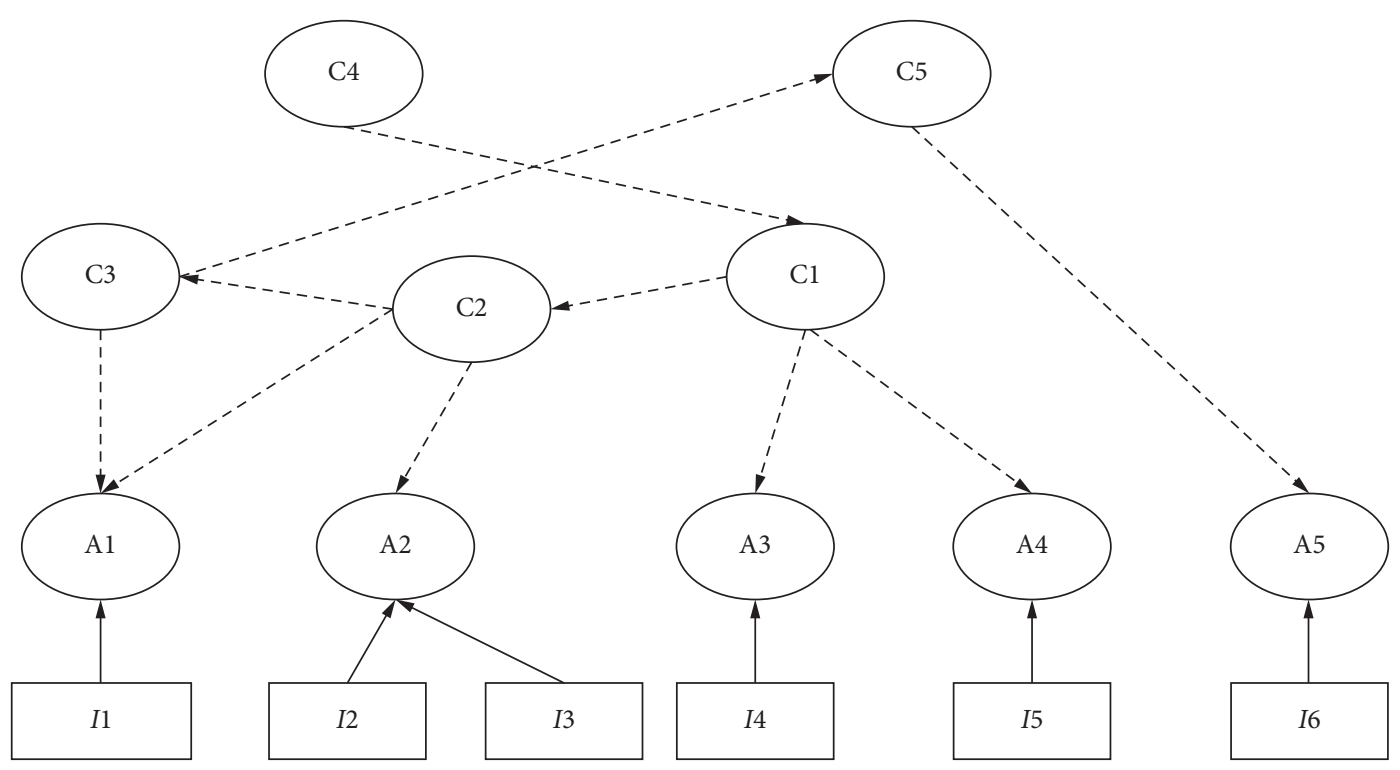

Figure 3: Tree structure of the association rule mapping model.

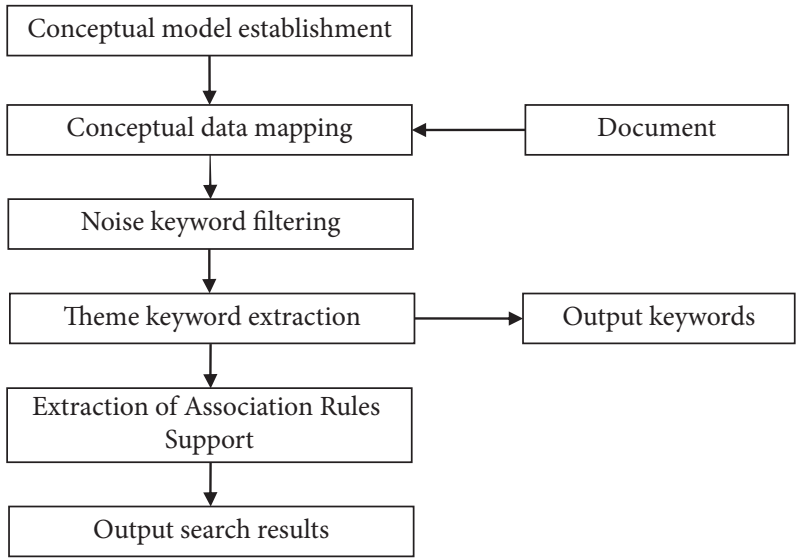

FIGURE 4: The retrieval model.

so that people can understand the traditional culture in a better and more complete way, and avoid the defects of information transmission. For example, some foreigners are very curious about traditional cultures such as hanfu and Chinese kung fu, but often these people only stay on the surface when they come into contact with these traditional cultures and do not have a deep understanding. The short video platform can give these foreigners the opportunity to understand Chinese traditional culture and make complex things as vivid as possible. For example, there will be various types of special cuisine and handicrafts on Douyin, plus the combination of some of the abovementioned music has given traditional culture more agility, and more and more foreigners have begun to appreciate the beauty of China's traditional culture. For example, the production process of Xi'an Roujiamo has been praised by many people. Some people's understanding of Xi'an may be from this short video. In the production process of the short video, it is necessary to select some essential content and the playing time of the short video. It is very short, so the producer must publish the essentials to quickly attract the attention of the viewers in a short time, otherwise the rough content may be directly ignored by the viewers.

3.2. The Form of Short Video Platform Has Freshness. In the actual process of newspaper advertising, the audience's participation is low. A short video can cause corresponding interactive. The user can navigate through the barrage, publish their views, and also find a corresponding short video author to communicate with the point of collision. This kind of instant participation can improve the quality of the content of the short video and also get the audience more involved in the short video creation. Through the integration of viewpoints, the traditional culture is further promoted and the concept is further innovated.

On the basis of traditional AR (augmented reality technology) and VR (virtual reality technology), MR (mixed reality technology) further establishes an interactive loop between the virtual world, the real world, and the user. This technology introduces real-world scene information into the virtual environment, and users can participate in all aspects of creation in real time during use and can apply for and join all the links they are interested in to meet the diverse needs of different people. In the project "Research on the Innovation of Confucian Cultural Expressions under the Leadership of MR Skill Tension," the focus is on the spread and application of traditional Chinese culture represented by Confucian culture under various new media technologies. At the beginning of the project research, reading a large number of domestic and foreign documents and Confucian classics, it can be seen that Confucian culture mostly uses text recording and inheritance, and there are few three-dimensional recording methods such as images and sounds. The text communication method has certain limitations in international communication. In the process of exporting, due to the lack of corresponding cultural heritage, many cultural 
recipients have no sense of cultural identity, which is not conducive to the inheritance and dissemination of Confucian culture and excellent Chinese culture. This is the socalled "cultural discount" phenomenon, that is, the contradiction between the localized culture and the foreign context, and the cultural discount leads to the weak output of the localized culture. Because people have different cultural backgrounds and cultural aesthetics, when reading a cultural work, the cognitive ability, understanding, and attractiveness will be greatly reduced due to different cultural backgrounds. In recent years, China's independent animation industry has developed rapidly, and a large number of excellent domestic animations have emerged, such as "Nezha" and "Big Fish Begonia," which have promoted the development and maturity of animation production methods. In the production of the short animation "Xiao Li Shuo," derived from the script of the project "The Son of the Plow," the script design was completed, and the design of the representative IP "Qu Xiao Li” of Qu Normal University was perfected, as well as various background designs and color designs. The animation completed the dubbing work and integrated into the short story animation "Xiao Li Shuo."

\subsection{Taking Advantage of the Social Features of Short Video} Platforms. Excellent traditional culture must attract more people's attention if it wants to be promoted. Short video platforms are very social and can spread quickly among people. Some short video platforms will have a lot of popular video below the user message. These people will communicate feelings after watching a short video and discuss the production of video content, and even some viewers will imitate some actions in the video. As a result, the popularity to make a short video will continue to increase. More and more video viewers and creators will be involved. The promotion of traditional culture will also be more extensive. Our government also has cooperation with many short video platforms, even on the trill platform which launched the government's official trill accounts. The government has also called for more and more people to participate in short video production, using advanced computer technology and new media technology to produce more high-quality short videos, promoting the development of China's traditional cultural heritage.

\subsection{Using Scientific and Technological Advantages to Improve} Image Quality. In short videos, the requirement for content quality is higher and need to be produced in a short time where the main body of a short video content is in line with the traditional culture; therefore, under the existing technical support, they can make use of existing technology to further simulation and display the charm of traditional culture, such as unmanned aerial vehicle (UAV) technology and VR/AR technology content quality improvement.

3.5. Innovating the Expression of Traditional Culture. The single propaganda method of traditional culture can no longer meet the needs of everyone. Therefore, it is necessary to further increase interesting culture in the process of traditional culture inheritance, improve the vitality of traditional culture, grasp the essence of traditional culture, and make full use of the existing science and technology to carry forward.

Most traditional cultures are traditional memories that people feel unfamiliar with, such as making bows and arrows. These methods are rarely seen in people's lives and young people know very little. Most young people even feel that these methods are useless and have little to do with their lives. At present, young people are still the main force of short video platforms. Most of the producers and viewers of short video platforms are young people. In order to spread traditional culture on short video platforms on a large scale, it must adapt to the aesthetics of young people. Video producers should pay attention to the trend of online popularity and add some fashion elements to the production of traditional cultural short videos. For example, some special effects or online language can be added to short videos to promote our country's traditions in a more modern and youthful way. By presenting the content of traditional culture in a more interesting way, only then can more and more people love traditional culture.

A high-quality short video must have a time control concept. The high-quality short video has a very strong ability to absorb information and can directly capture the consumer demand of the target consumer. However, most of the workers who promote traditional culture do not have the experience of learning professional photography and shooting skills. Many people shoot casually. The quality of many traditional cultural communication videos is very low, and there is no distinctive creative style, which cannot attract the audience. Although the threshold of many short video platforms is low, everyone can publish it with just one shot, but if there is no correct concept of user needs and the short video produced is not carefully designed, it is difficult to attract people's attention and it is impossible to produce high-quality works. Promoters who want to grasp the traditional cultural short video production skills in a short period of time must understand the communication characteristics of the short video platform and combine these characteristics to produce short videos. In the short video production process, various measures should be taken to attract viewers. It can form their own unique style, at the same time, short video producers can also hire some professional teams to operate their own short videos and enhance the vitality of short videos through cooperation.

Many short video platforms have opened a number of traditional cultural production accounts. These accounts rarely post content and have very few viewers. As a cultural institution, the government has the responsibility to promote the excellent local traditional culture and should take the lead in promoting the local traditional culture. The participation of official institutions can drive more people to participate in the army of traditional culture promotion. The government can also cooperate with some short video platforms and sign agreements. The two can make reasonable plans in the traditional propaganda process and plan some high-quality. The 


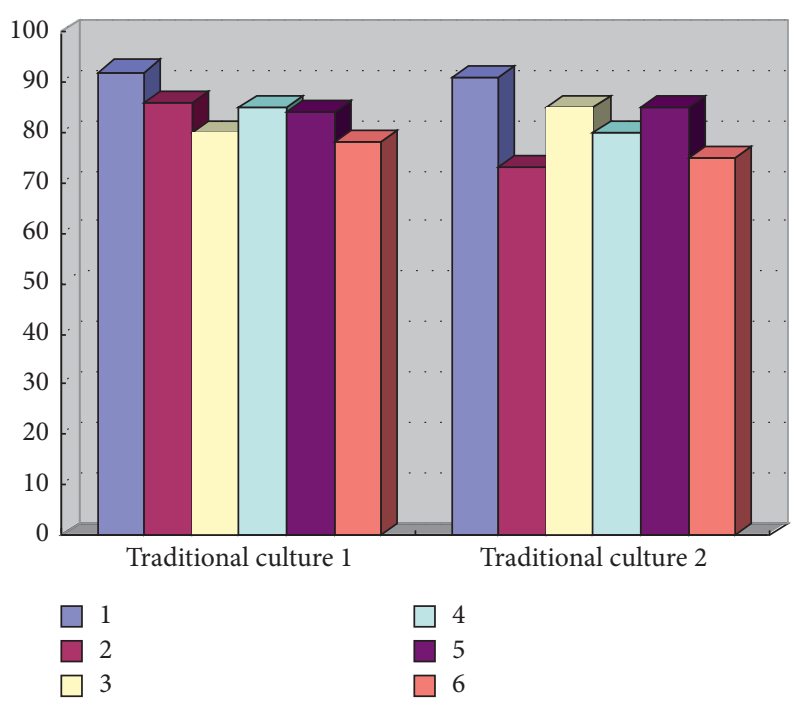

FIgURE 5: Evaluation of traditional culture.

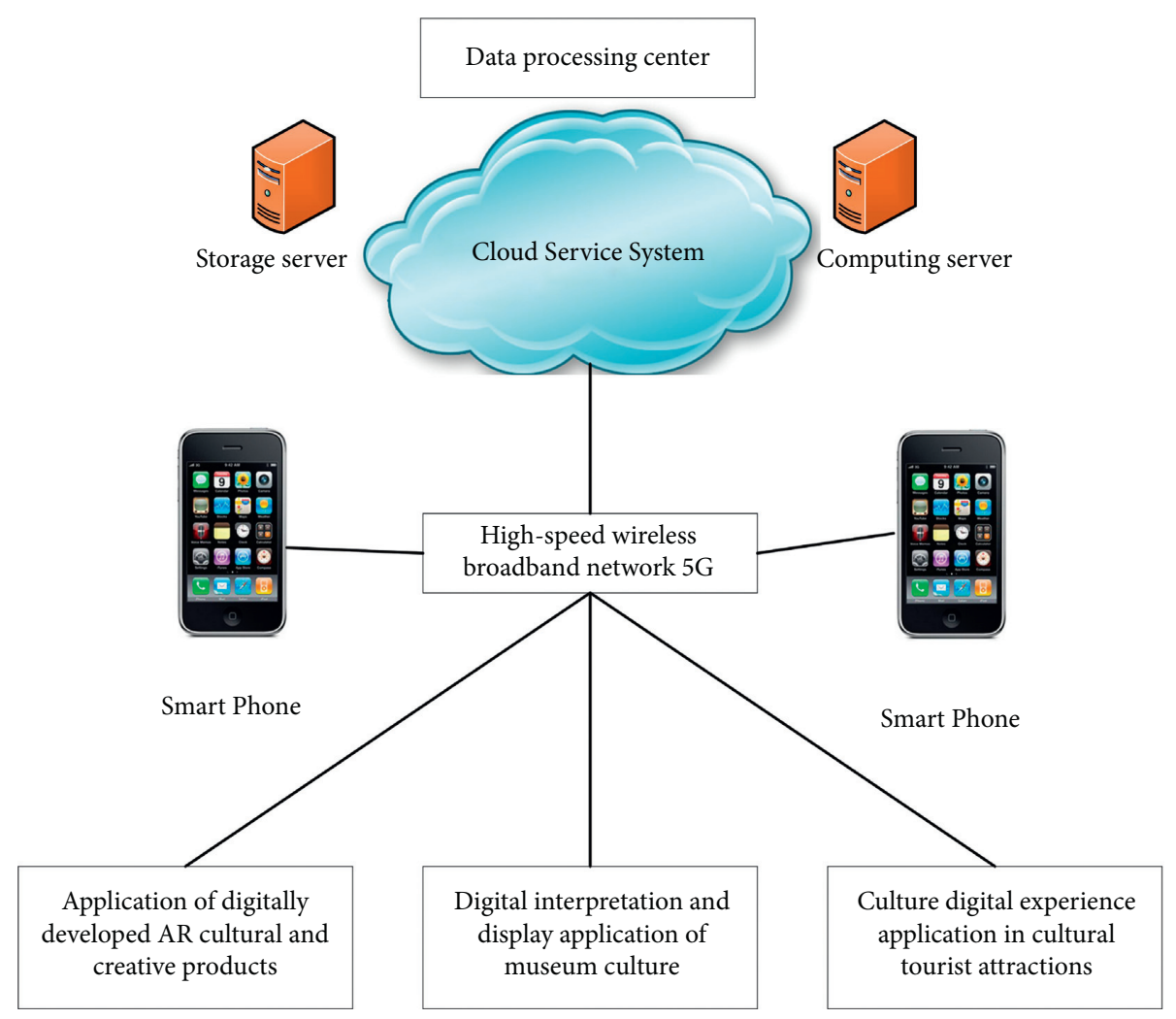

FIGURE 6: System hardware configuration network architecture.

government can hire some inheritors of intangible cultural heritage to join in the short publicity video, to provide a platform for the inheritance of traditional culture, and to call on more capable people to participate in it and contribute to the construction of the hometown. This new type of cooperation model is more conducive to the promotion of traditional culture, and it is also conducive to the establishment of a positive city image.

\section{Simulation Experiment}

How to evaluate the quality of the short video publicity system of traditional culture is also an important sign to test the effectiveness of short video publicity, especially for a large number of third-party evaluation data to carry out corresponding digital technology and data mining and fully extract and dig out the connection. 
4.1. Overview of Association Rule Mining Algorithms. For association analysis, it is necessary to delete and extract support by discovering the relationship hidden among the data and setting the corresponding confidence interval and the threshold value.

4.2. Data Acquisition and Preprocessing. Through the evaluation of 4000 traditional culture short video publicity systems as data sources, corresponding samples are selected, as shown in Figure 5, where each record represents a transaction. Correlation data mining analysis is to study the association analysis of multiple attributes and objects.

As can be seen from the evaluation of traditional culture in Figure 5, ideal results cannot be obtained directly if mining is carried out directly, and corresponding results need to be comprehensively expressed to achieve correlation analysis.

4.3. Correlation Analysis. After the corresponding data preprocessing and data cleaning, using the corresponding rules of decision tree analysis, initialization, setting up corresponding support (set to 0.25 ), the confidence level (set to 0.65 ), and the largest data set (3), through the corresponding rules mining, the specific process as shown in Figure 6.

Through the corresponding rules, it can be found that the corresponding Chinese-style songs have higher evaluation quality and more audiences like them, more than $85 \%$. For furniture with Chinese style, only $40 \%$ of people like it. Therefore, simulation experiments verify the effectiveness of data mining and digital technology.

Development of the traditional cultural products (based on AR technology) has the advantage of experience. A construct example of "cultural tourism products online experience exhibition center," focusing on display and showing that those based on AR technology are the development of cultural tourism products, using the experiential marketing strategy to carry out the experience type of promotional activities so as to attract tourists to buy (consumption) cultural tourism products.

Any AR data in the database are as follows:

$$
M_{i}=\left\{S_{1}, S_{2}, \ldots, S_{m s}\right\} \text {. }
$$

Sentence length sequence $L_{i}=\left\{L_{1}, L_{2}, \ldots, \mathrm{LMS}\right\}$. For the data fragment $G$ with $\mathrm{ml}$ length to be retrieved, the image fragment set $H$ of candidate AR is defined as follows:

$$
H=\left\{S_{i}+S_{i+1}+\cdots+S_{j}\left\|l_{i}+l_{i+1}+\cdots+l_{j}-m l\right\| \leq \varepsilon_{H} \quad(1 \leq i \leq j \leq k)\right\}
$$

Among them, $\varepsilon_{H}$ is the self-set length tolerance threshold, that is, the range of minimum length difference between two AR image fragments for comparison.

\section{Conclusions}

As archaeology, cultural relic protection and other fields receive more and more attention, the carrier of traditional culture is gradually favored by people, but how to carry out short video propaganda of traditional culture, so that more ordinary people receive more traditional culture is extremely important. By introducing digital technology, this paper tries to display traditional culture with modern technology. The data mining technology is used to sort out the content, publicity strategy, and publicity focus of traditional culture. Through correlation analysis, the audience of traditional culture is wider and the content has been transmitted more widely, aiming to make more Chinese people love traditional culture and promote traditional culture. The simulation results show that data mining and digital technology are effective and can effectively promote the propaganda of short videos of traditional culture.

\section{Data Availability}

Data sharing is not applicable to this article as no datasets were generated or analysed during the current study.

\section{Conflicts of Interest}

The authors declare that they have no conflicts of interest.

\section{Acknowledgments}

This work was supported by Cangzhou Normal University Campus Fund Project (no. xnjjw1912).

\section{References}

[1] K.-S. Yu, "A study on methods of traditional culture education for multi-cultural families by using video materials," The Studies of Korean Literature, vol. 55, no. 3, pp. 135-162, 2017.

[2] R. S. Jayawardena, W. Purahong, W. Zhang et al., "Biodiversity of fungi on Vitis vinifera L. revealed by traditional and high-resolution culture-independent approaches," Fungal Diversity, vol. 90, no. 1, pp. 1-84, 2018.

[3] K.-s. Yu and G.-s. Ahn, "A study on the method of traditional Korean culture course by using video materials-focusing on namseoul university cases," Journal of Language and Literature, vol. 62, no. 3, pp. 301-310, 2015.

[4] J. F. Van Lidth de Jeude, J. L. M. Vermeulen, P. S. Montenegro-Miranda, G. R. Van den Brink, and J. Heijmans, "A protocol for lentiviral transduction and downstream analysis of intestinal organoids," Journal of Visualized Experiments, no. 98, pp. 52531-52539, 2015.

[5] B. Zhou and A. Krott, "Data trimming procedure can eliminate bilingual cognitive advantage," Psychonomic Bulletin \& Review, vol. 23, no. 4, pp. 15-23, 2015. 
[6] L. A. Liikkanen and P. Åman, "Shuffling services: current trends in interacting with digital music," Interacting with Computers, vol. 28, no. 3, pp. 352-371, 2015.

[7] W. Dong, Q. Yang, Y. Liao et al., "Characterisation and comparison of the microflora of traditional and pure culture xiaoqu during the baijiu liquor brewing process," Journal of the Institute of Brewing, vol. 126, no. 2, pp. 1901-1910, 2020.

[8] I. E. Uwah, "The representation of african traditional religion and culture in Nigeria popular films," The Politics and Religion Journal-Serbian Edition, vol. 4, no. 1, pp. 1-7, 2017.

[9] I. Lyons, R. Hill, S. Deshong, G. Mooney, and G. Turpin, "Putting uncertainty under the cultural lens of traditional owners from the great barrier reef catchments," Regional Environmental Change, vol. 19, no. 6, pp. 1597-1610, 2019.

[10] D. Rose, D. Bell, and D. A. Crook, "Restoring habitat and cultural practice in Australia's oldest and largest traditional aquaculture system," Reviews in Fish Biology and Fisheries, vol. 3, no. 5, pp. 109-117, 2016.

[11] Y. Hu, S. Yu, S. Qin, D. Chen, J. Chu, and Y. Yang, "How to extract traditional cultural design elements from a set of images of cultural relics based on F-AHP and entropy," Multimedia Tools and Applications, vol. 80, no. 4, pp. 1-24, 2021.

[12] T. H. Lee, "A study of retirement culture in traditional ChinaSu shi's the ci poetry of fisherman," Studies in the Humanities, vol. 49, no. 5, pp. 165-172, 2016.

[13] C. W. Chang, T. S. Fu, D. Y. Lin et al., "Percutaneous balloon kyphoplasty and short instrumentation compared with traditional long instrumentation for thoracolumbar metastatic spinal cord compression," World Neurosurgery, vol. 130, no. 4, pp. e640-18, 2019.

[14] C. Ilaria, S. Giorgia, F. Piero et al., "Evaluation of autochthonous lactic acid bacteria as starter and non-starter cultures for the production of Traditional Mountain cheese," Food Research International, vol. 115, no. 3, pp. 6847-6854, 2018.

[15] R. Juppe, "Teaching English through short stories: organizing a literature: language course for non-traditional students," Bulletin of Tsukuba Gakuin University, vol. 7, no. 4, pp. 115-132, 2015.

[16] I. Meerschman, K. V. Lierde, C. V. Puyvelde, A. Bostyn, S. Claeys, and E. D'haeseleer, "Massed versus spaced practice in vocology: effect of a short-term intensive voice training versus a longer-term traditional voice training," International Journal of Language \& Communication Disorders, vol. 53, no. 2, pp. 197-207, 2018.

[17] L. R. Beuchat and D. A. Mann, "Comparison of new and traditional culture-dependent media for enumerating foodborne yeasts and molds," Journal of Food Protection, vol. 79, no. 1, pp. 95-111, 2016.

[18] Z. Peng, "Good gibbons and evil macaques: a historical review on cognitive features of non-human primates in Chinese traditional culture," Primates, vol. 56, no. 3, pp. 215-225, 2015. 